\title{
El Papel de la Fase de Observación de la Implementación en la Metodología Estudio De Clases
}

\author{
The Role of the Teaching and Observation Phases in the Lesson Study \\ Methodology
}

\author{
Adriana Breda ${ }^{*}$ \\ ORCID iD 0000-0002-7764-0511 \\ Viviane Hummes** \\ ORCID iD 0000-0003-2031-8238 \\ Rodrigo Sychocki da Silva*** \\ ORCID iD 0000-0002-7406-2517 \\ Alicia Sánchez ${ }^{* * * *}$ \\ ORCID iD 0000-0001-6569-6828
}

\begin{abstract}
Resumen
El objetivo de este trabajo es presentar el análisis detallado de la fase de implementación y contrastarlo con la fase de reflexión de una experiencia de Estudio de Clases. Para ello, con el uso de herramientas teóricas del Enfoque Ontosemiótico, se hizo el análisis didáctico de un video sobre un proceso de instrucción de geometría del espacio, en un contexto de un Estudio de Clases chileno. Los resultados muestran que el análisis detallado de la implementación de la clase permite observar aspectos relevantes que no fueron contemplados por el grupo de maestros en la fase de reflexión del Estudio de Clases. Se concluye que se debería dar un papel más importante a la fase de observación de la implementación en la metodología Estudio de Clases, por ejemplo, consensuando lo que se ha observado antes de pasar a la fase de reflexión.
\end{abstract}

Palabras-clave: Estudio de Clases. Enfoque Ontosemiótico. Configuración Didáctica. Geometría del Espacio.

\begin{abstract}
This work aims at presenting the detailed analysis of the implementation phase and contrast it with the reflection phase of a Lesson Study experience. To do this, we did a didactic analysis of a video about an instructional process about

\footnotetext{
* Doctora en Educación en Ciencias y Matemáticas por la Pontifícia Universidade Católica do Rio Grande do Sul (PUCRS). Docente e Investigadora en la Universidad de Barcelona (UB), Barcelona, España. E-mail: adriana.breda@ub.edu.

** Candidata a doctora por la Universidad de Barcelona (UB), Barcelona, España. E-mail: vhummes@ub.edu.

*** Doctor en Informática en la Educación por la Universidade Federal do Rio Grande do Sul (UFRGS). Docente e investigador en la Universidade Federal do Rio Grande do Sul (UFRGS), Rio Grande do Sul, Brasil. E-mail: rodrigo.sychocki@ufrgs.br

**** Candidata a doctora por la Universidad de Barcelona (UB), Barcelona, España. E-mail: asanchezb@ub.edu.
} 
space geometry in a context of a Lesson Study in Chile, using theoretical tools of the Onto-semiotic Approach. Results show that the lesson implementation detailed analysis allows us to observe relevant aspects that were not noticed by the group of teachers in the reflection phase of the Lesson Study. We conclude that the teaching and observation phases should be given a more important role in the Lesson Study methodology, for instance, reaching an agreement on what has been observed before starting the reflection phase.

Keywords: Lesson Study. Onto-semiotic Approach. Didactic Configuration. Geometry of Space.

\section{Aspectos Introductorios}

Una manera de ayudar a los profesores de matemáticas o a los maestros que enseñan matemáticas a mejorar su práctica docente consiste en potenciar la reflexión rigurosa y compartida sobre su práctica. La Lesson Study, en español Estudio de Clases (EC, a partir de ahora) es una metodología eficaz de formación de profesores que pretende ayudar a mejorar la práctica docente (FERNÁNDEZ; YOSHIDA, 2004; ISODA; ARCAVI; LORCA, 2007). El EC tiene una doble finalidad, por una parte, es un método orientado al cambio con el objetivo de mejorar la práctica docente y, por otra parte, está dirigido a la investigación para lograr una mejor comprensión de las prácticas (BALDIN, 2009; BURGHES; ROBINSON, 2010; PONTE et al., 2012).

La investigación sobre la práctica realizada en el marco del EC forma parte de una agenda de investigación más amplia sobre la reflexión del profesor como un aspecto clave para el perfeccionamiento de su práctica, la cual, a su vez, se enmarca en la investigación sobre el conocimiento y práctica profesional del profesor.

La relevancia que tiene el estudio de la práctica del profesor ha llevado, por una parte, a la generación de modelos teóricos de conocimientos y competencias del profesor de matemáticas, y, por otra parte, al desarrollo de modelos de análisis de la práctica del profesor (COLL; SÁNCHEZ, 2008) y, más en general, de los procesos de instrucción realizados por el profesor. Uno de ellos es el modelo de análisis didáctico formulado por el Enfoque Ontosemiótico de la Cognición e Instrucción Matemática (EOS, a partir de ahora) (FONT; PLANAS; GODINO, 2010; GODINO; BATANERO; FONT, 2007, 2019), el cual ha desarrollado herramientas de análisis específicas que ayudan a la descripción, explicación y valoración de los procesos de instrucción, y se ha aplicado al estudio de procesos de instrucción realizados en diferentes contextos (BADILLO et al., 2013; FONT; PLANAS, GODINO, 2010; GODINO; CONTRERAS; FONT, 2006; MATEUS, 2016; POCHULU; FONT; RODRÍGUEZ, 2016; VANEGAS; FONT; PINO-FAN, 2019). 
Dado el interés actual por investigar el EC y que, en la revisión realizada de la literatura no se ha encontrado un modelo teórico específico para el análisis de los procesos de instrucción realizados siguiendo esta metodología, resulta relevante preguntarse ¿qué conocimientos sobre el EC puede aportar el análisis minucioso y detallado de un proceso de instrucción, implementado con esta metodología, realizado con un modelo de análisis didáctico holístico de los procesos de instrucción como es el propuesto por el EOS?

El trabajo que aquí se presenta pretende responder a esta pregunta. Se trata de una investigación que forma parte de otra, más general, cuyo objetivo es realizar un estudio de caso para analizar, mediante el modelo de análisis didáctico propuesto por el EOS, las fases de planificación, implementación y reflexión de una experiencia de una clase de geometría del espacio desarrollada con la metodología Estudio de Clases en Chile.

El análisis de la fase de planificación y reflexión de este EC, usando la herramienta Criterio de Idoneidad Didáctica (CI) del EOS, se halla en Hummes, Breda y Seckel (2019), Hummes, Breda, Font y Sánchez (2020) y Hummes, Breda, Seckel y Font (2020). Los resultados obtenidos muestran cómo en las etapas de planificación y reflexión van apareciendo, en las reflexiones de los participantes, de manera implícita, algunos de los componentes e indicadores de los CI. Además, se observó que algunos de los CI, sus componentes e indicadores estaban presentes en la fase de planificación de dos maneras diferentes.

En algún caso, se hallaban en las orientaciones dadas sobre cómo realizar el EC y, en otros casos, aparecían en las reflexiones de los maestros al discutir la planificación, mientras que en la fase de reflexión se inferían de manera explícita, o implícita, en sus comentarios. Ahora bien, también se concluyó que las reflexiones de los maestros se hacían sobre descripciones que no coincidían con lo que los investigadores observaban en la implementación. Es decir, nuestro análisis de la implementación ponía en cuestión uno de los supuestos implícitos básicos de la metodología del EC.

En efecto, esta metodología se basa en el supuesto de que la reflexión se puede hacer a partir de lo observado en la implementación, sin necesidad de describir con mucho detalle lo que sucedió en ella. En cierta manera, se puede considerar que se infravalora la necesidad de describir previamente, en detalle, aquello sobre lo que se va a reflexionar (lo observado), ya que implícitamente se supone que la visión de la implementación de la clase proporciona toda la 
información necesaria (y de manera muy detallada) al profesor para poder realizar su reflexión compartida.

Las consideraciones anteriores nos llevaron a plantearnos como objetivo hacer un análisis detallado, con las herramientas que ofrece el modelo de análisis didáctico del EOS, de la implementación de la clase realizada en el marco de este EC y triangularlo con la fase de reflexión realizada por el grupo. Se trata de ver si la radiografía obtenida muestra aspectos relevantes no considerados, o bien, contradictorios con la valoración realizada por los maestros en la fase de reflexión.

Después de esta introducción, en la primera sección se presenta el marco teórico usado: la metodología EC y el modelo de análisis didáctico formulado por el EOS. En la segunda sección, se presenta la metodología cualitativa usada en la investigación. En la tercera sección, se hace el análisis detallado de la fase de implementación del EC, triangulándolo con los comentarios de los maestros en la fase de reflexión del EC. Por último, se presentan las reflexiones finales.

\section{Marco Teórico}

\subsection{Estudio de Clases}

La metodología EC surgió en Japón, como estrategia de desarrollo profesional docente, y se focaliza en el aprendizaje colectivo a partir de actitudes investigativas de la práctica de enseñanza. Consiste en el diseño colaborativo y detallado de una clase, de su implementación y observación directa en el aula, y de un análisis conjunto posterior (FERNÁNDEZ; YOSHIDA, 2004; LEWIS, 2002; MURATA, 2011; WANG-IVERSON; YOSHIDA, 2005).

La idea es que un grupo de profesores y especialistas se reúnan con una problemática en común sobre el aprendizaje de sus alumnos, planeen una lección para que el alumno aprenda, y, por último, examinen y discutan lo que ellos observan en dicha implementación. Existen diferentes modelos de ciclos de EC. La Figura 1 ilustra un ejemplo de un ciclo de EC que tiene lugar en Japón, y considera las siguientes etapas: 




Figura 1 - Ciclo de un EC

Fuente: adaptación de Murata (2011)

Los trabajos de Utimura, Souza Borelli y Curi (2020) muestran que algunos países del mundo occidental han contribuido para la diseminación de los EC, siendo Chile, desde el año de 2006, uno de ellos (OLFOS; ISODA; ESTRELLA, 2020). Lorca (2007) explica que en Chile se ha adaptado la metodología EC de manera que la preparación de la clase se basa en un problema identificado por los maestros y en las pautas de enseñanza que se encuentran en los libros de texto (primera y segunda etapa de un ciclo de EC).

Para esto, hay una selección de materiales con los que trabajar en la sesión de estudio. Tras preparar la lección, un maestro del grupo pone en práctica lo planeado (tercera etapa del ciclo). Los maestros, futuros profesores y el investigador pueden participar en la clase, observando y haciendo registros que sean significativos para ellos y que merecen ser discutidos en la sección de revisión y reflexión de la clase implementada (cuarta etapa del ciclo). En la reflexión se discuten las observaciones que cada participante hizo sobre la clase; se hacen preguntas sobre los problemas presentados en el aula, sobre la práctica del profesor, sobre el aprendizaje de los alumnos, verificando si lo implementado se acerca a los objetivos previstos. La clase puede prepararse nuevamente y luego volver a aplicarse, alimentando todo el proceso del EC (quinta etapa del ciclo) (ISODA; ARCAVI; LORCA, 2007).

En esta descripción de la metodología EC en Chile, queda claro que no se dan herramientas 
teóricas específicas para realizar la observación de la implementación, más allá de algunos criterios muy generales. Es decir, los participantes observan en base a su propio conocimiento.

\subsection{Modelo de análisis didáctico propuesto por el EOS}

El EOS (FONT; PLANAS; GODINO, 2010), considera cinco tipos de análisis sobre los procesos de instrucción: 1) Identificación de prácticas matemáticas; 2) elaboración de las configuraciones de objetos y procesos matemáticos; 3) análisis de las trayectorias e interacciones didácticas; 4) identificación del sistema de normas y metanormas; y 5) valoración de la idoneidad didáctica del proceso de instrucción.

El primer tipo de análisis explora las prácticas matemáticas realizadas en un proceso de instrucción matemático. El segundo se centra en los objetos y procesos matemáticos que intervienen en la realización de las prácticas, así como los que emergen de ellas. El tercer tipo de análisis didáctico está orientado, sobre todo, a la descripción de los patrones de interacción (LEGUIZAMÓN, 2017a, 2017b), las configuraciones didácticas y su articulación secuencial en trayectorias didácticas. El cuarto tipo de análisis se focaliza en las normas que regulan el proceso de instrucción (GODINO et al., 2009). El quinto tipo se basa en los cuatro análisis previos y está orientado a la identificación de mejoras potenciales del proceso de instrucción en nuevas implementaciones.

A continuación, explicamos las herramientas teóricas que se han generado en el EOS para realizar los cuatro primeros tipos de análisis didáctico. Por cuestiones de espacio no comentamos la noción de idoneidad didáctica (BREDA, 2020; BREDA; FONT; PINO-FAN, 2018; BREDA; LIMA, 2016; GIACOMONE; GODINO; BELTRÁN-PELLICER, 2018).

En el EOS se asume que la actividad matemática tiene como objetivo la resolución de tareas. Como resultado de un proceso de problematización, el sujeto, o la institución, asume resolver un problema, realizando, para ello, prácticas matemáticas. Para su realización y para la interpretación de sus resultados como válidos, se necesita, además del problema, poner en funcionamiento otros objetos matemáticos.

En la resolución es necesario el uso de lenguajes (verbales, simbólicos etc.), que son la parte ostensiva de una serie de definiciones, proposiciones y procedimientos que intervienen en la elaboración de los argumentos que permiten resolver el problema. En consecuencia, cuando un 
sujeto realiza y evalúa una secuencia de prácticas matemáticas, activa un conglomerado formado por situaciones-problemas, lenguajes, definiciones, proposiciones, procedimientos y argumentos, articulados en lo que, en términos del EOS, se llama una configuración de objetos primarios (FONT; GODINO; GALLARDO, 2013).

En el EOS, la noción de juego de lenguaje ocupa un lugar importante, al considerarla, junto con la noción de institución, como los elementos contextuales que relativizan las maneras de estar y de existir de los objetos matemáticos. Los objetos matemáticos intervienen en las prácticas matemáticas y emergen de las mismas, según el juego de lenguaje en que participan y se agrupan en las distintas dualidades (FONT; GODINO; GALLARDO, 2013):

$\checkmark$ Extensivo-intensivo: los objetos matemáticos pueden estar participando como particulares, o bien, como generales y, según el juego de lenguaje, pueden pasar de ser particulares a generales o viceversa.

$\checkmark$ Expresión-contenido: los objetos matemáticos pueden estar participando como representaciones, o bien, como objetos representados y, según el juego de lenguaje, pueden pasar de ser representaciones a ser objetos representados o viceversa.

$\checkmark$ Personal-institucional: los objetos matemáticos pueden estar participando como objetos personales, o bien, como objetos institucionales y, según el juego de lenguaje, pueden pasar de ser personales a ser institucionales. La dialéctica personal-institucional es esencial en los procesos de instrucción, ya que en ellos se pretende que los alumnos se apropien de los objetos institucionales (aprendan).

$\checkmark$ Ostensivo-no ostensivo: estos dos modos de estar de los objetos matemáticos en la práctica matemática se han de tomar como algo que se puede mostrar a otro directamente versus algo no se puede mostrar directamente, solamente por medio de otro algo, que sí se puede mostrar directamente. Los ostensivos matemáticos presentan una característica que es propia de las cosas del mundo real, que es la existencia real en el tiempo y en el espacio, mientras que a los objetos no ostensivos no se les atribuye este tipo de existencia, pues usualmente se considera que tienen una existencia ideal.

$\checkmark$ Unitario-sistémico: cuando una entidad matemática es considerada como un objeto, se está adoptando una perspectiva unitaria sobre el mismo. Ahora bien, hay momentos en que interesa adoptar una perspectiva sistémica sobre dicho objeto, por ejemplo, considerando 
las partes que lo componen. En esta dualidad, los objetos matemáticos pueden estar participando como objetos unitarios, o bien, como un sistema.

La resolución de problemas se realiza mediante la articulación de secuencias de prácticas. Tales secuencias tienen lugar en el tiempo y se suelen considerar, en muchos casos, como procesos. En particular, el uso y/o la emergencia de los objetos primarios de la configuración (problemas, definiciones, proposiciones, procedimientos y argumentos), tiene lugar mediante los respectivos procesos matemáticos de comunicación, problematización, definición, enunciación, elaboración de procedimientos (creación de algoritmos y rutinas) y argumentación (aplicando la dualidad procesoproducto). Por otra parte, las dualidades antes descritas, dan lugar a los siguientes procesos: institucionalización - personalización, generalización - particularización, análisis/descomposición - síntesis/reificación, materialización/concreción - $\quad$ idealización/abstracción, expresión/representación - significación.

Una noción clave para relacionar las prácticas con los objetos primarios y los procesos activados en ellas es la noción de función semiótica (FS). En el EOS, las FS se conciben, de manera metafórica, como una correspondencia entre conjuntos que pone en juego tres componentes: un plano de expresión (objeto inicial); un plano de contenido (objeto final); un criterio o regla de correspondencia (GODINO; BATANERO; FONT, 2007, 2019).

La FS se relaciona, de entrada, con la dualidad expresión/contenido y con el proceso de significación; por ejemplo, cuando a alguien se le pregunta qué entiende por triángulo (expresión) y responde que es un polígono de tres lados (contenido). Pero en el EOS se amplía esta manera de entender la FS y se considera que, tanto el plano de la expresión como el del contenido, pueden ser objetos materiales o mentales, que pueden ser cualquiera de los seis tipos de objetos primarios de la configuración y, además, cualquiera de las maneras de estar participando en la práctica matemática consideradas en las dualidades, por ejemplo, la FS que relaciona una propiedad con otra (la FS que relaciona el teorema de Pitágoras con el teorema de Pitágoras generalizado, por ejemplo), al contemplar la dualidad extensivo-intensivo, la podemos convertir, si es el caso, también en una FS que relaciona un particular (un extensivo) con un general (un intensivo). En este trabajo entendemos las FS como un instrumento relacional entre los objetos primarios activados en las prácticas matemáticas. La noción de función semiótica es más general que la noción de conexión tal como se usa en la teoría extendida de las conexiones (TEC), ya que los tipos de 
conexiones contempladas en este marco teórico se consideran casos particulares de funciones semióticas (RODRÍGUEZ-NIETO et al., 2021).

En el EOS, la noción de configuración didáctica (CD) constituye la principal herramienta metodológica para el análisis a nivel micro de los procesos de instrucción (GODINO; CONTRERAS; FONT, 2006). Se define como cualquier segmento de actividad didáctica (enseñanza y aprendizaje) comprendido entre el inicio y fin del proceso de resolución de una tarea. Incluye, por tanto, las acciones de los estudiantes y del profesor, así como los medios planificados o usados para abordar la tarea.

Se consideran cuatro tipos de configuraciones teóricas. La configuración adidáctica se concreta en una secuencia de situaciones adidácticas de acción, formulación, validación, y la situación didáctica de institucionalización. La manera tradicional o clásica de enseñar matemáticas basada en la presentación magistral, seguida de ejercicios de aplicación caracteriza la configuración magistral. Cuando la resolución de la tarea se realiza por el estudiante sin una intervención directa del docente (por ejemplo, resolver ejercicios propuestos por el profesor fuera del aula) se trata de un tipo de configuración didáctica en la que básicamente predomina el estudio personal. Otro tipo de configuración puede definirse respetando el momento de exploración por parte de los alumnos, pero asumiendo el profesor la validación y la institucionalización mediante un diálogo contextualizado entre el docente y los alumnos. Las configuraciones didácticas empíricas que acontecen están más o menos próximas a estas configuraciones teóricas.

En el EOS, se considera que la metodología para investigar los procesos de instrucción consiste en realizar el análisis de la configuración didáctica y de la trayectoria didáctica (secuencia de configuraciones didácticas) y también que, tanto las prácticas matemáticas como el proceso de instrucción, están regulados, entre otras, por normas epistémicas (regulan las prácticas y contenidos matemáticos, en correspondencia con el discurso matemático que es posible desarrollar en una institución), normas mediacionales (regulan los recursos - temporales o artefactos - utilizados en las prácticas), normas interaccionales (regulan los modos de interacción entre las personas involucradas en la práctica o proceso instructivo matemático), normas ecológicas (aspectos externos -directrices curriculares, políticas educativas- que condicionan las prácticas del aula) (GODINO; BATANERO; FONT, 2019).

Dada la gran diversidad de interacciones didácticas ocurridas en cualquier proceso de instrucción, a veces conviene centrarse en las interacciones en torno a conflictos de tipo semiótico. 
De acuerdo con Godino, Batanero y Font (2007) entendemos por conflicto semiótico (CS) cualquier disparidad entre los significados atribuidos a una expresión por dos sujetos, personas o instituciones. Cuando el profesor lo utiliza como estrategia didáctica con la intención de crear una contradicción en el alumno acerca de las prácticas que ha realizado; hablamos de CS de tipo cognitivo, puesto que la disparidad se produce entre prácticas de un mismo sujeto). Cuando se produce un conflicto, por ejemplo, entre el mundo de las matemáticas y el aula de matemáticas o entre el aula de matemáticas y el mundo cotidiano, la disparidad se produce entre prácticas propias de instituciones diferentes. En este caso, hablamos de CS de tipo epistémico.

En toda CD se puede diferenciar tres componentes: a) una configuración epistémica (prácticas, objetos, FS y procesos matemáticos institucionales requeridos en la tarea), b) una configuración instruccional (sistema de funciones docentes, discentes y medios instruccionales que se utilizan, así como las interacciones entre los distintos componentes y las normas que las regulan) y c) una configuración cognitiva - afectiva (sistema de prácticas, objetos y procesos matemáticos personales que describe el aprendizaje y los componentes afectivos que lo acompañan, así como los CS que pueden dificultar el aprendizaje).

Esto da origen a la siguiente problemática que es la que nos ha interesado en esta investigación: ¿Qué conocimientos sobre el EC puede aportar el análisis minucioso y detallado de un proceso de instrucción, implementado con esta metodología, realizado con un modelo de análisis didáctico holístico de los procesos de instrucción como es el propuesto por el EOS?

\section{Metodología}

Se realiza el análisis cualitativo de una parte de un video que presenta diferentes etapas de un EC. Se trata de un estudio de caso (YIN, 2001), donde mediante el modelo de análisis didáctico propuesto por el EOS, se analiza la fase de implementación de una experiencia de una clase de geometría del espacio desarrollada en el contexto de la metodología EC en Chile. El video es producto del Centro de Perfeccionamiento, Experimentación e Investigaciones Pedagógicas del Ministerio de Educación de Chile y fue publicado en la plataforma YouTube en el 24 de enero de 2012. Se puede accederlo mediante el link: https://www.youtube.com/watch?v=VUPTkKJ8ij8\&t=1165s. Después de una búsqueda de 
diferentes videos sobre EC en la plataforma YouTube, se seleccionó este por el importante número de visualizaciones y, porque se puede observar el ciclo de EC completo.

El video tiene tres partes: En la primera, los maestros se reúnen para planificar colectivamente una clase de matemáticas basada en un estándar de desempeño del currículo chileno para nivel básico 2: a) Caracterizan prismas rectos y pirámides considerando el número y la forma de las caras y el número de aristas y vértices; b) Seleccionan redes de prismas y pirámides para armar un cuerpo geométrico dadas algunas características de éste. En la segunda, un profesor que participó de la planificación implementa la clase con los alumnos de nivel básico 2 (duración aproximada de 10 minutos ya que algunos segmentos de la implementación se han recortado). En la tercera, el colectivo de maestros hace una reflexión, poniendo atención en cómo los alumnos y alumnas reaccionaron en el aula, cómo expresaron sus ideas y cómo el profesor condujo el proceso de aprendizaje.

El análisis del video se divide en las siguientes etapas. En la primera etapa se hace una transcripción de la implementación de la clase evidenciada en el video. En la segunda se segmenta la transcripción en partes que se considera que corresponden a una configuración didáctica (CD), es decir un segmento de actividad de enseñanza y aprendizaje que se distribuye entre los momentos de inicio y finalización de una tarea implementada. Aunque el criterio básico para determinar un segmento de transcripción correspondiente a una CD es el intervalo que va desde el inicio hasta la finalización de una tarea implementada, la agrupación en configuraciones didácticas es flexible y queda a la discreción del investigador. El proceso de enseñanza de un contenido matemático tiene lugar en un determinado tiempo que incluye una secuencia de CD (trayectoria didáctica).

En la tercera etapa, para cada segmento de transcripción se determina la CD. En esta investigación, para cada $\mathrm{CD}$, se han identificado las prácticas matemáticas, los procesos y los objetos primarios activados en ellas (tareas, representaciones, proposiciones, definiciones, procedimientos y argumentos), las funciones semióticas (FS) que debe establecer el alumno para dar significado a la actividad matemática realizada, las funciones/acciones del profesor y de los alumnos, el tipo de CD, los CS, los patrones de interacción y las normas. En la cuarta etapa se triangularon los hechos didácticos significativos que los investigadores observaron en el análisis de las CD con las reflexiones que hicieron los participantes del EC sobre la implementación de la clase.

A continuación, en esta sección se presenta un ejemplo de cómo se desarrollaron estas 
etapas. Primero, se presentan la Figura 2 y las líneas de la transcripción del inicio de la clase implementada las cuales se considera que se corresponden con la CD1. A continuación, se detalla la CD1 (Cuadro 1) y también la trama de FS (Figura 3) contemplada en esta CD. Por último, se triangula con los comentarios del grupo de maestros en la fase de reflexión del EC.

\section{a) Transcripción correspondiente a la CD1}

Profesor: Vamos a recordar lo que son los vértices y las aristas. ¿Ok?

Alumnos: Ok.

Profesor: Ya. Entonces vamos primero a observar este cuerpo que tenemos acá ¿Ya? Y

Ustedes me van a decir: ¿cuántas aristas tenemos acá? (CPEIP, 2012, s/p)

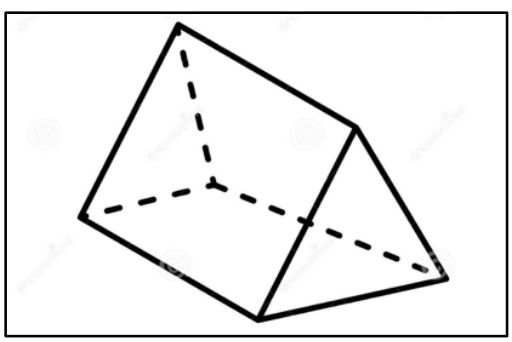

Figura 2 - Prisma que el profesor muestra a los alumnos Fuente: adaptado de CPEIP (2012)

b) Configuración Didáctica CD1 


\begin{tabular}{|c|c|c|c|c|c|c|c|c|c|}
\hline Prácticas & Procesos & Objetos & FS & $\begin{array}{c}\text { Funciones } \\
\text { del docente }\end{array}$ & $\begin{array}{c}\text { Funciones del } \\
\text { alumno }\end{array}$ & $\begin{array}{c}\text { Tipo de } \\
\text { Configuración }\end{array}$ & CS & $\begin{array}{l}\text { Patrones de } \\
\text { interacción }\end{array}$ & Normas \\
\hline $\begin{array}{l}\text { Práctica 2: Contar } \\
\text { el número de } \\
\text { aristas, sobre } \\
\text { representaciones/ } \\
\text { modelos } \\
\text { tridimensionales }\end{array}$ & 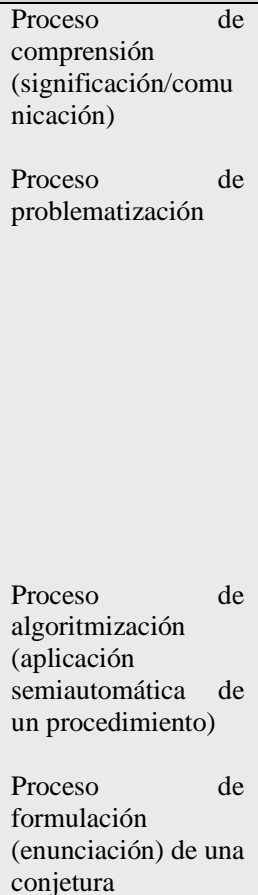 & 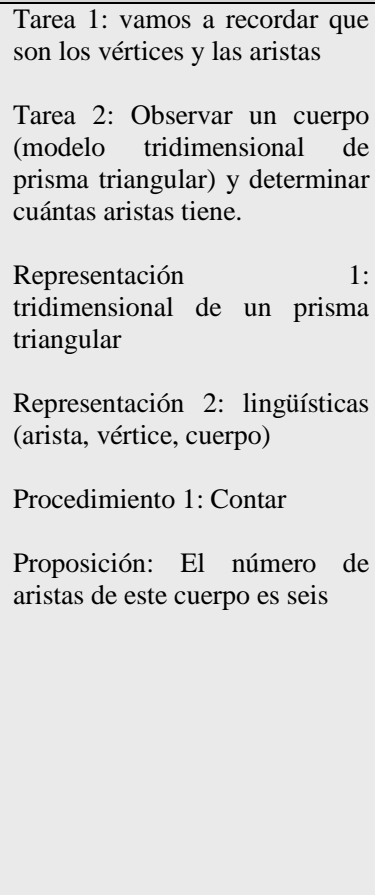 & $\begin{array}{l}\text { De la FS1 a } \\
\text { la FS10 } \\
\text { FS11 } \\
\text { FS12 y } 13\end{array}$ & $\begin{array}{l}\text { Presentar la } \\
\text { tarea }\end{array}$ & $\begin{array}{l}\text { Resolver la tarea } \\
\\
\text { Responder la } \\
\text { pregunta }\end{array}$ & $\begin{array}{l}\text { Adidáctica/Personal } \\
\text { /Grupal }\end{array}$ & $\begin{array}{l}\text { No se } \\
\text { observa }\end{array}$ & $\begin{array}{l}\text { No hay } \\
\text { evaluación } \\
\text { directa por parte } \\
\text { del profesor } \\
\text { sobre el proceso } \\
\text { de interacción: } \\
\text { Pregunta del } \\
\text { profesor - } \\
\text { Respuesta de los } \\
\text { alumnos. }\end{array}$ & $\begin{array}{l}\text { Hay que } \\
\text { intentar } \\
\text { resolver } \\
\text { las tareas } \\
\text { que } \\
\text { propone } \\
\text { el } \\
\text { profesor y } \\
\text { contestarl } \\
\text { as }\end{array}$ \\
\hline
\end{tabular}

Cuadro 1 - Configuración Didáctica (CD1)

Fuente: los autores 
Dado que las configuraciones didácticas se inician normalmente con una tarea, los dos primeros procesos (comprensión y problematización) están presentes en casi todas las $\mathrm{CD}$, por más simple que sea la tarea, ya que los alumnos deben entender lo que se les pregunta y asumirlo como algo que tienen que resolver. Por otra parte, el tipo de configuración se considera de tipo Adidáctica/Personal/Grupal porque el profesor presenta la tarea, los alumnos se responsabilizan de resolverla y no hay ningún tipo de evaluación (ni de validación, ni de institucionalización) directa de la respuesta de los alumnos por parte del profesor (sí existe de manera indirecta al no cuestionar la respuesta dada por los alumnos), ni ninguna demanda del profesor para que los alumnos justifiquen su respuesta, ver Figura 3.

c) FS presentes en la CD1

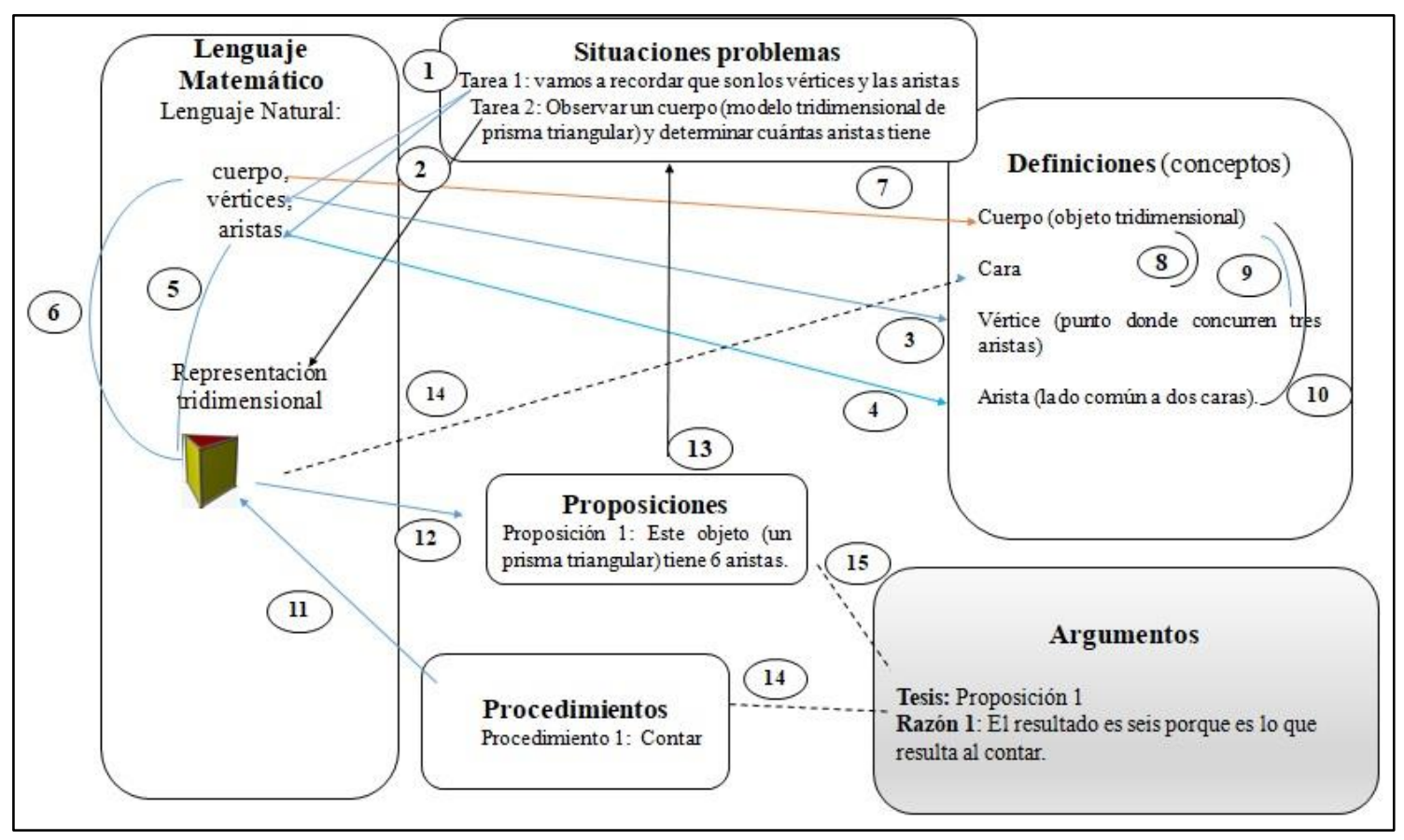

Figura 3 - FS presentes en la CD1

Fuente: los autores

En la Figura 3 se muestra la trama de FS que deben hacer los alumnos para dar significado a la actividad matemática realizada en la CD1. Por ejemplo, la FS1 relaciona el objeto primario tarea (expresión) con un objeto lingüístico (contenido) y la FS13 una proposición (expresión) con una tarea (contenido). Para entender las tareas, los alumnos deben relacionar los términos lingüísticos verbales y la representación tridimensional que aparecen en la tarea entre sí y también darles algún tipo de significado (FS1 hasta FS7). 
A su vez, estos significados (definiciones implícitas) se relacionan entre ellos (FS8 hasta FS10). Después, deben contar las aristas de la representación tridimensional (FS11) que les permite concluir que este cuerpo tiene seis aristas (FS12), con lo que se puede considerar resuelta la tarea (FS13). Además, hay una FS que sería importante establecer, la que relaciona la representación tridimensional con la noción de cara de un prisma y dos que están implícitas que se podrían haber establecido claramente si el profesor hubiese preguntado cómo estaban seguros de que la respuesta era seis (FS14 y FS15) (representadas en la Figura 3 por líneas discontinuas y con el contenido de la FS en color gris).

d) Triangulación del análisis de la implementación, referente a la CD1, con la fase de reflexión del EC

No se observaron en la fase de reflexión de los maestros comentarios sobre la CD1, aunque hay algunos hechos en esta $\mathrm{CD}$ que resultan muy significativos para los investigadores. El primero es que el profesor no evalúa explícitamente el resultado que dan los alumnos (lo hace indirectamente al no cuestionar la respuesta dada por los alumnos, con lo que valida e institucionaliza al mismo tiempo la respuesta dada), el segundo es que no les pide que argumenten su respuesta. Teniendo en cuenta que el objetivo que había planificado el grupo de maestros era el descubrimiento de una relación entre el número de lados de la base de una pirámide y el número de vértices y de aristas, se podría aprovechar esta CD1 para hacerles observar que habían encontrado la respuesta contando los vértices del cuerpo que el profesor presentó, y después, preguntar si también contarían en el caso de un cuerpo que tuviese 105600 vértices. La limitación del procedimiento de contar directamente se podría utilizar como una buena razón para buscar el número de vértices y aristas sin tener que contar directamente (objetivo planificado).

\section{Resultados}

Como primer resultado del análisis detallado de la transcripción de la clase implementada, hemos encontrado que ella se segmenta en $16 \mathrm{CD}$, conforme se presenta en el Cuadro 2:

\begin{tabular}{|c|l|}
\hline Configuración Didáctica (CD) & \multicolumn{1}{c|}{ Descripción } \\
\hline CD1 & Determinar el número de aristas de un prisma triangular. \\
\hline CD2 & Determinar cuál es la pirámide cuya base tiene el menor número de lados posible. \\
\hline CD3 & $\begin{array}{l}\text { Presentación de la tarea: construir pirámides de diferentes bases, calcular el } \\
\text { número de vértices y de aristas y completar la fila correspondiente de una tabla de }\end{array}$ \\
\hline
\end{tabular}




\begin{tabular}{|c|c|}
\hline & $\begin{array}{l}\text { tres columnas (número de lados de la base, número de vértices y número de } \\
\text { aristas). }\end{array}$ \\
\hline CD4 & Construcción de la pirámide triangular. \\
\hline CD5 & $\begin{array}{l}\text { Discusión con un grupo de alumnos que había construido una pirámide de base un } \\
\text { cuadrado, sobre cuántos lados de la base, aristas y vértices había que quitar para } \\
\text { obtener una pirámide de base triangular. }\end{array}$ \\
\hline CD6 & $\begin{array}{l}\text { Contar las aristas y los vértices de la pirámide de base triangular y completar la } \\
\text { fila correspondiente de la tabla de la CD3. }\end{array}$ \\
\hline CD7 & Construcción de la pirámide cuya base es un cuadrado. \\
\hline CD8 & $\begin{array}{l}\text { Iniciar la tarea de contar las aristas y los vértices de la pirámide de base } \\
\text { cuadrangular y completar la fila de una tabla de tres columnas (número de lados } \\
\text { de la base, número de vértices y número de aristas). Como un alumno confunde el } \\
\text { número de aristas con los lados de la base, el profesor no termina esta CD y pasa } \\
\text { a la siguiente. }\end{array}$ \\
\hline CD9 & $\begin{array}{l}\text { Discutir qué pirámide se podría construir con una base de cuatro lados que no fuese } \\
\text { un cuadrado. }\end{array}$ \\
\hline CD10 & Terminar la CD8 (No se ve en el vídeo). \\
\hline CD11 & Construcción de la pirámide cuya base es un hexágono. \\
\hline CD12 & $\begin{array}{l}\text { Contar las aristas y los vértices de la pirámide de base hexagonal y completar la } \\
\text { fila correspondiente de la tabla (No se ve en el vídeo). }\end{array}$ \\
\hline CD13 & Construcción de la pirámide cuya base es un heptágono (No se ve en el vídeo). \\
\hline CD14 & $\begin{array}{l}\text { Contar las aristas y los vértices de la pirámide de base heptagonal y completar la } \\
\text { fila correspondiente de la tabla. }\end{array}$ \\
\hline CD15 & $\begin{array}{l}\text { Encontrar una regularidad recursiva que permite obtener el número de vértices y } \\
\text { de aristas de una pirámide a partir del número de vértices y de aristas de una } \\
\text { pirámide cuya base tiene un lado menos. }\end{array}$ \\
\hline CD16 & $\begin{array}{l}\text { Encontrar una relación entre el número de lados de la base de una pirámide y el } \\
\text { número de vértices y de aristas. }\end{array}$ \\
\hline
\end{tabular}

Cuadro 2 - Trayectoria de CD

Fuente: los autores

Para cada una de las CD hemos seguido las etapas explicadas en la metodología. Por cuestiones de espació, en esta sección presentamos la configuración didáctica CD15 (Cuadro 3), la cual se corresponde con el momento donde el profesor conduce a los estudiantes a encontrar una regularidad entre el número de lados de la base de una pirámide y el número de vértices y el número de aristas, que hemos considerado la más crucial de la implementación y, también, una de las más ricas en términos de análisis didáctico. En la siguiente sección se realiza una discusión global sobre los resultados obtenidos del análisis didáctico de todas las CD.

\section{a) Transcripción correspondiente a la CD15}

Profesor: La base tres tiene una cantidad de vértice y una cantidad de aristas, ¿cierto? Y así sucesivamente las otras bases. Quiero que pongan bien atención qué está sucediendo en relación a la base y a los demás datos, ¿ya?

Alumnos: Sí.

Profesor: Entonces voy a hacer la siguiente pregunta: Aquí en la base cinco que yo deje un vacío [apunta a la tabla en la pizarra] cuántas (...) sin hacer este cuerpo, sino que, solamente ya usando nuestra idea, ¿cierto? Nuestro pensamiento (CPEIP, 2012, s/p). 


\begin{tabular}{|c|c|c|c|c|c|c|c|c|c|}
\hline Prácticas & Procesos & Objetos & FS & $\begin{array}{c}\text { Funciones del } \\
\text { docente }\end{array}$ & $\begin{array}{c}\text { Funciones } \\
\text { del alumno }\end{array}$ & $\begin{array}{c}\text { Tipo de } \\
\text { Configuración } \\
\end{array}$ & CS & $\begin{array}{l}\text { Patrones de } \\
\text { interacción }\end{array}$ & Normas \\
\hline $\begin{array}{l}\text { Práctica 4: } \\
\text { Encontrar } \\
\text { una } \\
\text { regularidad }\end{array}$ & $\begin{array}{l}\text { Proceso de } \\
\text { comprensión } \\
\text { /significació } \\
\text { n/comunicac } \\
\text { ión } \\
\text { Proceso de } \\
\text { problematiza } \\
\text { ción. } \\
\text { Proceso de } \\
\text { formulación/ } \\
\text { enunciación } \\
\text { de una } \\
\text { conjetura }\end{array}$ & $\begin{array}{l}\text { Tarea: Solo observando la tabla, sin construir la } \\
\text { pirámide, determinar el número de vértices y de } \\
\text { aristas de la pirámide de base pentagonal. Completar } \\
\text { la fila correspondiente de la tabla } \\
\text { Representación: Tabular con las casillas } \\
\text { correspondientes al número de vértices y aristas de } \\
\text { línea de la pirámide de base pentagonal vacías } \\
\text { Procedimiento: Encontrar una regularidad recursiva } \\
\text { analizando la variación entre un término y el anterior } \\
\text { Proposición 1: El número de vértices de la pirámide } \\
\text { de base pentagonal es } 6 \text { y el de aristas } 10 \\
\text { Proposición 2: los números de los vértices van en } \\
\text { orden (una proposición que establece una regularidad } \\
\text { de tipo recursiva: el número de vértices de una } \\
\text { pirámide se obtiene sumando uno al número de } \\
\text { vértices de la pirámide cuya base es un polígono con } \\
\text { un número de lados inferior en una unidad). } \\
\text { Proposición 3: los números de las aristas van saltando } \\
\text { de dos en dos (una proposición que establece una } \\
\text { regularidad de tipo recursiva: el número de aristas de } \\
\text { una pirámide se obtiene sumando dos al número de } \\
\text { aristas de la pirámide cuya base es un polígono con } \\
\text { un número de lados inferior en una unidad). } \\
\text { Argumento 1: he observado que los números de los } \\
\text { vértices están ordenados, el siguiente de } 5 \text { es } 6 \text {. } \\
\text { Argumento 2: he observado que los números de las } \\
\text { aristas van saltando, el anterior es } 8 \text { y sumándole dos } \\
\text { se tiene 10. }\end{array}$ & $\begin{array}{l}\text { De la } \\
\text { FS9 a la } \\
\text { FS } 14\end{array}$ & 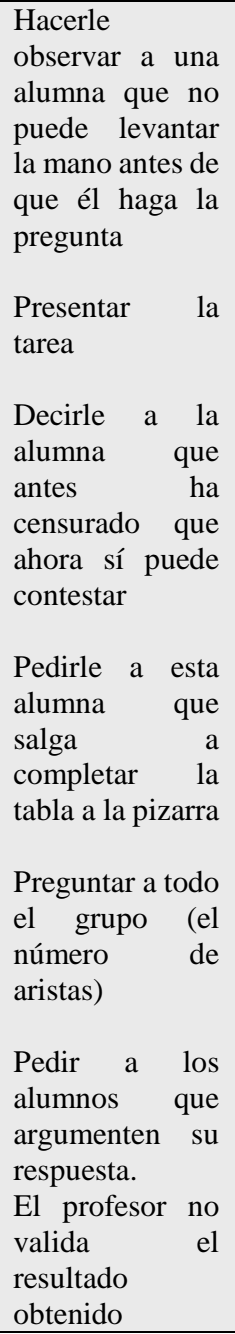 & 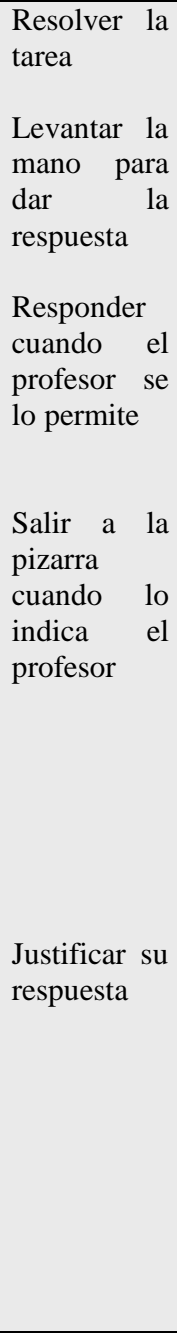 & Dialógica & $\begin{array}{l}\text { CS1: en la } \\
\text { casilla de } \\
\text { la } \\
\text { cartulina } \\
\text { correspon } \\
\text { diente al } \\
\text { número de } \\
\text { lados de la } \\
\text { base, el } \\
\text { profesor } \\
\text { ha escrito } \\
\text { base } 5\end{array}$ & $\begin{array}{lr}\text { No } & \text { hay } \\
\text { evaluación directa } \\
\text { por parte del } \\
\text { profesor sobre el } \\
\text { proceso } \\
\text { interacción: } \\
\begin{array}{lr}\text { Pregunta } \\
\text { profesor }\end{array} \\
\begin{array}{lr}\text { Respuesta de } \\
\text { alumnos. }\end{array}\end{array}$ & $\begin{array}{l}\text { Se debe } \\
\text { responder sin } \\
\text { usar el modelo } \\
\text { tridimensional } \\
\\
\text { No se debe } \\
\text { levantar la } \\
\text { mano antes de } \\
\text { que el } \\
\text { profesor haga } \\
\text { la pregunta } \\
\\
\text { Se sale a la } \\
\text { pizarra } \\
\text { cuando lo } \\
\text { indica } \\
\text { profesor }\end{array}$ \\
\hline
\end{tabular}

Cuadro 3 - Configuración Didáctica (CD15)

Fuente: los autores 
La CD15 empieza con la tarea de determinar el número de vértices y aristas de una pirámide de base pentagonal, solamente observando una tabla (Figura 4).

\begin{tabular}{|ccc|}
\hline $\begin{array}{c}\text { Número de lados } \\
\text { de la base de la } \\
\text { pirámide }\end{array}$ & $\begin{array}{c}\text { Número de } \\
\text { vértices }\end{array}$ & $\begin{array}{c}\text { Numero de } \\
\text { aristas }\end{array}$ \\
\hline Base 3 & 4 & 6 \\
\hline Base 4 & 5 & 8 \\
\hline Base 5 & & \\
\hline Base 6 & 7 & 12 \\
\hline Base 7 & 8 & 14 \\
\hline
\end{tabular}

Figura 4 - Imagen de la cartulina empleada por el profesor en la clase Fuente: Adaptado de CPEIP (2012)

Las prácticas matemáticas que se ponen en juego a partir de esta tarea son que los alumnos encuentren una regularidad y que la argumenten. También se ponen en juego los procesos de comprensión, problematización, formulación y argumentación. Por otra parte, el tipo de configuración se considera de tipo dialógica, porque el profesor presenta la tarea y va conduciendo a los alumnos, por medio de un diálogo de preguntas y respuestas justificadas a la solución de la tarea, sin embargo, no hay ningún tipo de evaluación directa de la respuesta de los alumnos por parte del profesor, dado que, al final, él acaba conduciendo a los alumnos a la regularidad que buscaba conseguir CD16. También se evidencia un potencial Conflicto Semiótico (CS1) por parte del profesor, cuando escribe en la cartulina base 5 para referirse a una base con número de lados igual a 5 .

b) FS que el alumno debe realizar para dar significado a la actividad matemática de la CD15

En el análisis de la CD15 hemos encontrado 14 FS (Figura 5) que los alumnos deben realizar para dar sentido a la actividad matemática realizada. 


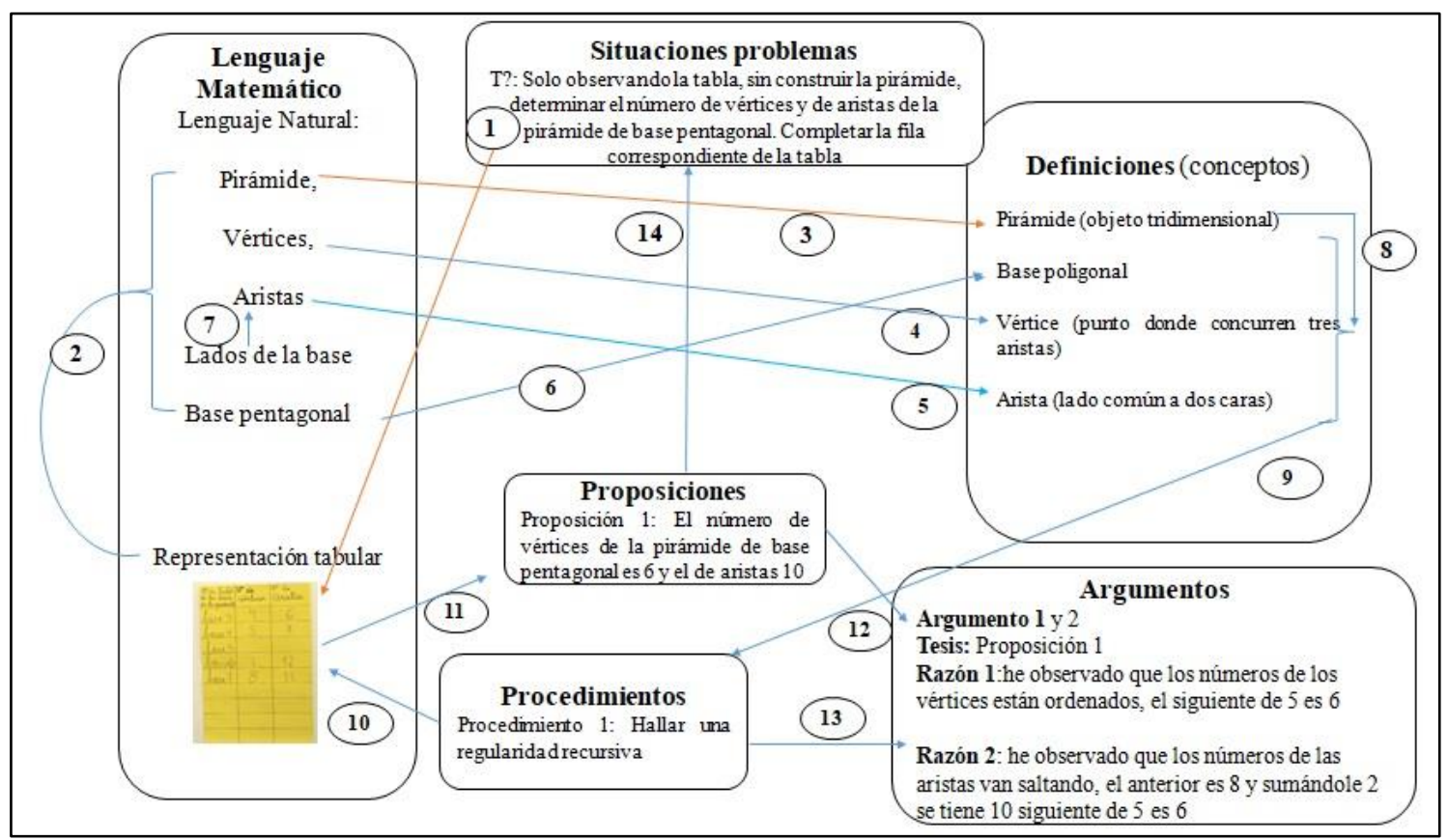

Figura 5 - FS presentes en la CD15

Fuente: los autores

La FS1 relaciona el objeto primario tarea (expresión) con un objeto lingüístico (representación tabular), la FS2 relaciona la representación tabular con los términos lingüísticos de la primera fila de la tabla, a los cuales se les debe dar algún tipo de significado (de la FS3 a la FS7). Estas FS relacionan el objeto lenguaje natural Pirámide, Vértices, Aristas, lados, y tipo de base con las definiciones de Pirámide, Vértice, Arista, y base poligonal, respectivamente. Para resolver la tarea, la alumna debe encontrar la regularidad (FS10) y establecer una conjetura (una proposición para ella) (FS11) usando los significados de los términos que aparecen en la tabla (FS8 y FS9) y, también, ante la demanda del profesor tiene que argumentar por qué su conjetura es cierta (FS12 y FS13).

c) Fase de reflexión sobre la implementación de la CD15

En la siguiente reflexión los maestros tienen en cuenta cómo los alumnos reaccionaron y cómo el profesor condujo el proceso de aprendizaje. Los maestros se denominan por M1, M2, M3 y M4 y el profesor (P) que implementó la clase.

M2: Como dijo la maestra M4, en cierto momento los chiquillos vieron de forma vertical la tabla. M4: Sí.

M2: La lectura fue de forma vertical. ¡Ya! ¿Y el objetivo nuestro, cuál era? 
P: El objetivo de nosotros era que fuera horizontal. Hacer que los niños supiesen que a la base solamente tendrían que sumar uno para saber cuántos vértices y que el doble siempre va a ser las aristas.

M2: Cuando P hace alusión a la base cinco y una alumna instantáneamente empieza a levantar la mano, antes que él haga la pregunta, ¿esperaba que los chiquillos dieron respuestas tan rápidamente, como surgió en el video?

P: No, creo que, cuando lo planificamos, si recuerdan, tuvimos algunas dudas con respecto a si iban a ser capaces pronto de llegar a esto, ¿cierto? De responder a esos espacios que habiamos dejado dentro de esa tabla que hicimos. Y no pensé que iba ser tan rápido.

M4: Yo la verdad creo que la acción de la alumna evidencia claramente que los niños ahí en ese minuto abandonaban lo concreto y se fueron al abstracto. Que en realidad es el objetivo final de una clase de matemática. Que salten y que, no construyó ella la pirámide 5, si no que ella saltó inmediatamente a lo mental.

M1: Ahora yo pienso que el vehículo aquí fundamental ¿no es cierto? en la consecución de los resultados y de que la alumna respondiera tan rápido fue la tabla. [Apunta para la tabla] Esta tabla que nosotros planteamos cuando hicimos la planificación, fue la que le dio el indicador clarísimo para lograr establecer ya un orden meramente abstracto, o sea, trabajar en forma numérica (CPEIP, 2012, s/p).

\section{d) Triangulación de la CD15 con la fase de reflexión del EC}

Los maestros, en el momento de la reflexión, no observaron un conflicto semiótico (CS) potencial causado por el hecho de que profesor ponga, por ejemplo, base 3 para indicar el número de lados de la base.

Además, la discusión de los maestros sobre la mirada vertical y horizontal de la tabla se podría precisar si se tienen en cuenta las proposiciones, procedimientos y argumentos de la CD15. Por ejemplo, la mirada vertical se podría precisar como regularidad recursiva, es decir, la recursividad se encuentra al ver que conforme aumentan los lados de la base, aumenta en una unidad el número de vértices $(4,5,6$ etc.) y las aristas aumentan de dos en dos $(6,8,10$, etc.), y la mirada horizontal, como dos proposiciones sobre la relación entre número de lados de la base de una pirámide y el número de vértices y de aristas.

La reflexión introducida por M4 sobre la evidencia del abandono de lo concreto para ir a lo abstracto se puede precisar mucho más si se tiene en cuenta que lo que hay es la conjetura de una regularidad y que el hecho de no construir la pirámide era una condición del problema impuesta por el profesor (norma). 


\subsection{Discusión global}

El objetivo planificado era encontrar una relación entre el número de lados de la base de una pirámide y el número de vértices y de aristas. En la CD1 (Cuadro 1) el profesor pretende recordar los conocimientos previos de arista y vértice, pero genera un CS de tipo epistémico ya que en lugar de hablar del número de lados de la base habla de base tres, base cuatro, etc., durante toda la implementación de la clase. La existencia de este CS se observa desde la planificación de la clase

$P:$ (...) tenemos el número base de la pirámide (mientras lo escribe en una pizarra)

M3: El número de lados de la base

M4: Claro (CPEIP, 2012, s/p).

En la CD1, el profesor no valida directamente la respuesta de los alumnos ni les pide que justifiquen su respuesta, razón por la cual se ha caracterizado como Adidáctica/Personal/Grupal. Teniendo en cuenta que el objetivo que había planificado el grupo de maestros era el descubrimiento de una relación entre lados de la base, vértices y aristas, la limitación del procedimiento de contar directamente se podría utilizar como una buena razón para buscar el número de vértices y aristas sin tener que contar directamente (objetivo planificado).

En la CD15 (Cuadro 3) los alumnos encuentran el número de vértices y de aristas de la pirámide de base pentagonal, y ante las demandas de justificación del profesor argumentan que han encontrado una regularidad recursiva que les permite determinar que los números de las aristas van saltando de 2 en 2 y el de vértices de 1 en 1 . Se trata de una CD cuasi dialógica, ya que el profesor no institucionaliza el resultado obtenido primero y, después, no hace ver la dificultad de utilizar esta regularidad para conocer el número de aristas y de vértices de una pirámide de base un polígono de muchos lados, lo cual daría sentido a buscar una relación entre en número de lados de la base y el número de aristas y vértices, que era el objetivo planificado. Lo que hace el profesor es gestionar, a continuación, una CD16 de tipo dialógico en la que él dirige directamente a los alumnos a encontrar la regularidad que él quería, de acuerdo con la planificación realizada. Los alumnos llegan a encontrar que el número de vértices es el número de lados de la base más uno y el número de aristas el doble del número de lados de la base, también usan la relación de manera inversa, dado el número de vértices hallan el número de lados y el de aristas.

Si se compara el análisis detallado de la trayectoria de las CD (Cuadro 2) con las valoraciones del grupo de maestros, se destaca que no se comentan los CS de tipo epistémico que ha generado el profesor. Por ejemplo, en la CD9 el profesor pregunta qué pirámide se podría 
construir con una base de cuatro lados que no fuese un cuadrado. La interacción que gestiona el profesor consigue que los alumnos le respondan que puede ser un rectángulo y él valida e institucionaliza esta respuesta. En esta CD9 se produce un CS de tipo epistémico, ya que los alumnos tenían pajitas de la misma longitud, por lo que la base siempre era un polígono regular (es decir solo se podría construir una pirámide de base rectangular si se suponía que las pajitas podían representar aristas de longitudes diferentes, lo cual no se dice en ningún momento).

Tampoco hay reflexiones sobre cómo el profesor ha gestionado en particular la CD1 y la CD15 ya que podría haber aprovechado las respuestas de los alumnos para justificar la necesidad de buscar la regularidad que era el objetivo planificado. También, es significativo que en la reflexión comenten la interacción del profesor con la alumna que ha salido a responder la CD15, pero no lo interpretan en términos de establecimiento, por parte del profesor, de normas de cómo intervenir en la clase.

Otro aspecto a destacar es que los maestros hacen comentarios en sus reflexiones sobre lo particular y lo general y el papel del material concreto para facilitar el tránsito entre ellos, pero son comentarios que se podrían precisar mucho si se tienen en cuenta las prácticas, procesos, proposiciones, procedimientos y argumentos explicitados en la trayectoria de CD. Lo mismo sucede con sus comentarios sobre el trabajo colaborativo y cómo se ha gestionado la interacción de la clase.

\section{Consideraciones finales}

Con el cotejo entre el análisis detallado de la fase de implementación y la fase de reflexión de un EC sobre geometría del espacio en el contexto chileno, se concluye, principalmente, que el paso de acordar, entre los participantes del EC, lo que pasó en la implementación, es un paso al que no se da, en esta metodología, la importancia que tiene. Por ejemplo, en la reflexión de los maestros no aparece ningún comentario acerca de los CS de tipo epistémico que comete el profesor. Es decir, lo que se observa es que los maestros observan y reflexionan en base a su conocimiento y éste puede ser diferente de un maestro a otro y, en muchos casos, puede ser un conocimiento que no permite observar ciertos hechos que se han producido.

La variedad de enfoques teóricos en Educación Matemática (EM) ofrece lentes diferentes, que permiten ver ciertos hechos, regularidades y explicaciones, de orden micro y macro didáctico, 
que son difíciles de observar sin este enfoque teórico, sin embargo, no hay ningún enfoque que se pueda considerar paradigmático en el área de la Educación Matemática, por tanto, puede haber tantas descripciones como enfoques teóricos diferentes se usen para hacer el análisis de la implementación de la clase. Por ejemplo, la descripción minuciosa de lo que sucedió, por medio del uso de las herramientas del EOS, sería muy útil para concretar y dirigir la reflexión del grupo hacía aspectos a los que no le han dado importancia y que pueden ser muy relevantes para un análisis y un rediseño del proceso de instrucción.

Esta diversidad de enfoques, en el caso de querer mejorar la competencia de observación, descripción y análisis de la implementación de los participantes en un EC mediante el uso de algún marco teórico, conlleva primero el problema de qué enfoque elegir y, segundo, cómo se enseñan a los profesores que participan en un EC los constructos del enfoque teórico que se quieren usar como lentes para hacer la descripción de la implementación.

Eso nos hace reflexionar que lo que es un hecho en el área de la EM es difícil de caracterizar, ya que los hechos son observados por un observador con ciertos lentes teóricos que, al observarlos, los convierte en fenómenos. Sin embargo, si asumimos la idea del hecho como la parte de un fenómeno sobre la cual hay consenso, lo que se sugiere en este trabajo es que esta fase de consensuar lo que se ha observado debería tener un papel más relevante en la metodología EC de lo que, en nuestra opinión, tiene. Consideramos que disponer de una observación consensuada intermedia entra la visión directa del vídeo o presencial de la clase y la reflexión del profesor mejoraría y ampliaría dicha reflexión.

\section{Agradecimientos}

Trabajo desarrollado en el marco del proyecto de investigación PGC2018-098603-B-I00 (MCIU/AEI/FEDER, UE), en el contracto del Programa Juan de la Cierva Formación (FJCI-201734021) y en el marco del programa de Doctorado Pleno en el Exterior proceso número 88881.173616/2018-01 (Capes).

\section{Referencias}

BADILLO, E.; FIGUEIRAS, L.; FONT, V.; MARTÍNEZ, M. Visualización gráfica y análisis comparativo de la práctica matemática en el aula. Enseñanza de las Ciencias, Barcelona, v. 31, n. 3, p. 207-225, 2013. 
BALDIN, Y. Y. O significado da introdução da metodologia japonesa de Lesson Study nos cursos de capacitação de professores de matemática no Brasil. En: ENCONTRO ANUAL DA SBPN E SIMPÓSIO BRASIL-JAPÃO, 18., 2009, São Paulo. Anais do XVIII Encontro Anual da SBPN e Simpósio BrasilJapão. São Paulo: SBPN, 2009. p. 01-09.

BREDA, A. Características del análisis didáctico realizado por profesores para justificar la mejora en la enseñanza de las matemáticas. Bolema, Rio Claro, v. 34, n. 66, 2020, p. 69-88.

BREDA, A.; FONT, V.; PINO-FAN, L. R. Criterios valorativos y normativos en la Didáctica de las Matemáticas: el caso del constructo idoneidad didáctica. Bolema, Rio Claro, v. 32, n. 60, 2018, p. 255278.

BREDA, A.; LIMA, V. M. R. Estudio de caso sobre el análisis didáctico realizado en un trabajo final de un máster para profesores de matemáticas en servicio. REDIMAT - Journal of Research in

Mathematics Education, Barcelona, v. 5, n. 1, 2016, p. 74-103.

BURGHES, D. N.; ROBINSON, D. Lesson study: enhancing mathematics teaching and learning. Plymouth: CfBT Education Trust, 2010.

COLL, C.; SÁNCHEZ, E. Presentación. El análisis de la interacción alumno-profesor: líneas de investigación. Revista de Educación, Madrid, v. 346, p. 15-32, 2008.

CPEIP. Estudio de Clases: Geometría Centro de Perfeccionamiento, Experimentación e Investigaciones Pedagógicas del Ministerio de Educación de Chile. 2012. Disponible en: https://www.youtube.com/watch?v=VUPTkKJ8ij8. Acceso: 12 mar. 2021.

FERNÁNDEZ, C.; YOSHIDA, M. Lesson study: a Japanese approach to improving mathematics teaching and learning. Mahwah: Erlbaum, 2004.

FONT, V.; GODINO, J. D.; GALLARDO, J. The emergence of objects from mathematical practices. Educational Studies in Mathematics, Utrecht, v. 82, n. 1, p. 97-124, 2013.

FONT, V.; PLANAS, N.; GODINO, J. D. Modelo para el análisis didáctico en educación matemática. Infancia y Aprendizaje, Madrid, v. 33, n. 1, p. 89-105, 2010.

GIACOMONE, B.; GODINO, J.; BELTRÁN-PELLICER, P. Desarrollo de la competencia de análisis de la idoneidad didáctica en futuros profesores de matemáticas. Educação e Pesquisa, São Paulo, v. 44, p. e172011, 2018.

GODINO, J. D.; BATANERO, C.; FONT, V. The onto-semiotic approach to research in mathematics education. ZDM, Berlín, v. 39, n. 1-2, p. 127-135, 2007.

GODINO, J. D.; BATANERO, C.; FONT, V. The onto-semiotic approach: implications for the prescriptive character of didactics. For the Learning of Mathematics, Edmonton, v. 39, n. 1, p. 37-42, 2019.

GODINO, J. D.; CONTRERAS, A.; FONT, V. Análisis de procesos de instrucción basado en el enfoque ontológico-semiótico de la cognición matemática. Recherches en Didactiques des Mathematiques, Lyon, v. 26, n. 1, p. 39-88, 2006. 
GODINO, J. D. et al. Aproximación a la dimensión normativa en didáctica de las matemáticas desde un Enfoque Ontosemiótico. Enseñanza de las Ciencias, Barcelona, v. 27, n. 1, 2009, p. 59-76.

HUMMES, V. B.; BREDA, A.; SECKEL, M. J. Idoneidad didáctica en la reflexión de profesores: análisis de una experiencia de estudio de clases. En: MARBÁN, J. M.; ARCE, M.; MAROTO, A.; MUÑOZESCOLANO, J. M.; ALSINA, Á. (Ed.), INVESTIGACIÓN EN EDUCACIÓN MATEMÁTICA, 23., 2019. Actas del XXIII Simposio de la Sociedad Española de Investigación en Educación Matemática. Valladolid: SEIEM, 2019. p. 381-390.

HUMMES, V.; BREDA, A.; SÁNCHEZ, A.; FONT, V. Didactical Suitability Criteria in Videos of Lesson Study. Quaderni di Ricerca in Didattica, Palermo, v. 3, 2020, p. 257-268.

HUMMES, V. B.; BREDA, A.; SECKEL, M. J.; FONT, V. Criterios de Idoneidad Didáctica en una clase basada en el Lesson Study. Praxis \& Saber, Tunja, v. 11, n. 26, 2020, p. e10667.

ISODA, M.; ARCAVI, A.; LORCA, A. M. El estudio de clases japonés en matemáticas: Su importancia para el mejoramiento de los aprendizajes en el escenario global. Valparaíso: Ediciones Universitarias de Valparaíso/Pontificia Universidad Católica de Valparaíso, 2007.

LEGUIZAMÓN, F. Evolución de los patrones de interacción comunicativa de los docentes de matemáticas: Caso UPTC. 2017. Tesis (Doctorado en Ciencias de la Educación no publicada) Universidad Pedagógica y Tecnológica de Colombia, Tunja, 2017a.

LEGUIZAMÓN, J. F. Patrones de interacción comunicativa del profesor universitario de matemáticas: un estudio de caso. Praxis \& Saber, Tunja, v. 8, n. 16, p. 57-82, 2017 b.

LEWIS, C. C. Lesson study: A handbook of teacher-led instructional change. Philadelphia: Research for Better Schools, 2002.

LORCA, A. M. El estudio de clases japonés en perspectiva. En: JORNADAS NACIONALES DE EDUCACIÓN MATEMÁTICA, 13., 2007, Viña del Mar. Actas de la XIII Jornadas Nacionales de Educación Matemática. Viña del Mar: Sociedad Chilena de Educación Matemática, 2007. p. 1-7.

MATEUS, E. Análisis Didáctico a un Proceso de Instrucción del Método de Integración por Partes. Bolema, Rio Claro, v. 30, n. 55, p. 559-585, 2016.

MURATA, A. Introduction: conceptual overview of lesson study. En: HART, L. C.; ALSTON, A. S.; MURATA, A. (ed.). Lesson study research and practice in Mathematics Education. New York: Springer, 2011. p. 1-12.

OLFOS, R.; ISODA, M.; ESTRELLA, S. Más de una década de Estudio de Clases en Chile: hallazgos y avances. Paradigma, Maracay, v. 41, p. 190-221, 2020.

POCHULU, M.; FONT, V.; RODRÍGUEZ, M. Desarrollo de la competencia en análisis didáctico de formadores de futuros profesores de matemática a través del diseño de tareas. Revista Latinoamericana de Investigación en Matemática Educativa - RELIME, Ciudad de México, v. 19, n. 1, p. 71-98, 2016.

PONTE, J. P. D. et al. Aprendizagens profissionais dos professores de Matemática através dos estudos de aula. Perspectivas da Educação Matemática, Campo Grande, v. 1, n. 1, p. 7-24, 2012. 
RODRÍGUEZ-NIETO, C. et al. Mathematical connections from a networking of theories between Extended Theory of Mathematical Connections and Onto-semiotic Approach. International Journal of Mathematical Education in Science and Technology, London, 2021 (published online). Doi: https://doi.org/10.1080/0020739X.2021.1875071.

UTIMURA, G. Z.; SOUZA BORELLI, S. de; CURI, E. Lesson Study (Estudo de Aula) em diferentes países: uso, etapas, potencialidades e desafios. Educação Matemática Debate, Montes Claros, v. 4, p.1$16,2020$.

VANEGAS, Y.; FONT, V.; PINO-FAN, L. Análisis de la práctica profesional de un profesor cuando explica contenidos de medida. En: BADILLO, E.; CLIMENT, N.; FERNÁNDEZ, C.; GONZÁLEZ, M. T. (ed.). Investigación sobre el profesor de matemáticas: formación, práctica de aula, conocimiento y competencia profesional. Salamanca: Ediciones Universidad Salamanca, 2019. p. 43-62.

WANG-IVERSON, P.; YOSHIDA, M. Building our understanding of lesson study. Philadelphia: Research for Better Schools, 2005.

YIN, R. K. Estudo de caso: planejamento e métodos. Porto Alegre: Bookman, 2001.

Submetido em 30 de Junho de 2020. Aprovado em 08 de Outubro de 2020. 